\title{
Absence of masking in the path of apparent movement*
}

\author{
FRED ATTNEAVE and GENE BLOCK \\ University of Oregon, Eugene, Oregon 97403
}

\begin{abstract}
Alternation of diagonal pairs of lights produced apparent movement which $\mathbf{S}$ could organize in either of two distinctly different ways, as instructed. The task was to detect movement of a weak probe light that either was or was not located in the path of the apparent movement, depending on the organization, stimulation being identical in the two cases. Results showed no evidence of path-specific masking.
\end{abstract}

Under conditions promoting good apparent movernent, the impression is very strong that a determinate object is actually moving through a well-defined path, fleetingly occupying successive positions between the generating stimuli at successive instants. A naive but not necessarily incorrect hypothesis is that some process representing the object really does move through a representational medium under these conditions. There is convincing evidence (Attneave \& Block, 1973) that stimuli are mapped into an analog model of physical space before apparent movement between them occurs, but we do not know whether the apparent movement is or is not itself represented in an analog manner, i.e., by real movement within the model. If it is, we might reasonably expect it to have some measurable effect on the path traversed.

Masking is the most obvious such effect to look for. Kolers (1963) found that the detectability of a weak probe light was not impaired in the path of apparent movement. We were not convinced, however, that he looked for masking at an appropriate time. His probe was always presented between the movement-generating stimuli, in time as well as in space, whereas one could hardly expect apparent movement to occur, in real time, until after the presentation of both the stimuli that generate it. (The issue is complicated, however, by the fact that the probe was very dim, and may accordingly have had a long entry time.)

In the present experiment, therefore, we presented probe signals at various intervals after, as well as before, the onset of the second movement-generating stimulus, which was simultaneous with the offset of the first (whereas Kolers had probed for masking during a 105-msec ISI). At the same time, we investigated this further question: Even if apparent movement does not mask a light in its path, may it nevertheless mask a small movement, or displacement of a probe?

\section{METHOD}

\section{Subjects}

Two paid Ss, a male graduate student in psychology and a female undergraduate at the University of Oregon, served in the experiment.

*This research was supported by NIMH Grant MH 20 449-03, for "Studies on Spatial Representation."

\section{Apparatus}

The S viewed a $36-\mathrm{cm}-\mathrm{sq}$ display panel from a distance of $2.74 \mathrm{~m}$. The four main light sources were arranged in a square configuration, $10 \mathrm{~cm}$ apart, as shown in Fig. 1. Each was a bank of four red $(600 \mathrm{~m} \mu)$ LEDs (light-emitting diodes) behind a $1.26-\mathrm{cm}$ circular aperture. Covering the apertures and fronting the panel was a diffusing surface of tracing paper held flat by a rigid sheet of plastic. These lights had an intensity of about $.03 \mathrm{~cd}$.

The lights on opposite corners of the square were turned on and off simultaneously. When the two diagonal pairs were alternated, they produced an excellent appearance of movement, but the movement could be seen in either of two strikingly different ways: either (a) horizontally, as a top light moving in one direction (left vs right) and a bottom light moving in the other, or (b) vertically, as a left light and a right light moving up and down in opposite phase. With a little practice, both Ss were able to see the movement either horizontally or vertically at will; this voluntary control was essential to the design of the experiment.

There were two probe locations, one in the path between the upper left and upper right main lights at a distance of $3 \mathrm{~cm}$ from the latter, the other between the lower left and lower right lights, $3 \mathrm{~cm}$ from the former (see Fig. 1). At each of these locations, two LEDs were placed side by side as close as their structure allowed, about $3 \mathrm{~mm}$ between centers. The brightness

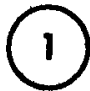

\section{PROBE}
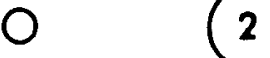
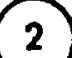
PROBE

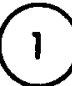

Fig. 1. 
Table 1

Proportion of Identifications Correct on Both Position and Direction of Probe Movement When Probe Was in Path of Apparent Movement (H) and When It Was Not (V)

\begin{tabular}{|c|c|c|c|c|c|c|c|c|c|}
\hline & \multirow{2}{*}{$\begin{array}{l}\text { Path of } \\
\text { Apparent } \\
\text { Movement }\end{array}$} & \multicolumn{7}{|c|}{ Time of Probe Movement } & \multirow[b]{2}{*}{ Mean } \\
\hline & & -120 & -60 & -30 & 0 & +30 & +60 & +120 & \\
\hline S D & $\begin{array}{l}\mathrm{V} \\
\mathrm{H} \\
\mathrm{V}-\mathrm{H}\end{array}$ & $\begin{array}{l}.641 \\
.625 \\
.016\end{array}$ & $\begin{array}{l}.703 \\
.625 \\
.078\end{array}$ & $\begin{array}{r}.594 \\
.641 \\
-.047\end{array}$ & $\begin{array}{l}.641 \\
.609 \\
.032\end{array}$ & $\begin{array}{r}.563 \\
.656 \\
-.093\end{array}$ & $\begin{array}{l}.609 \\
.563 \\
.046\end{array}$ & $\begin{array}{r}.609 \\
.641 \\
-.032\end{array}$ & $\begin{array}{l}.623 \\
.623 \\
.000\end{array}$ \\
\hline S I & $\begin{array}{l}\mathrm{V} \\
\mathrm{H} \\
\mathrm{V}-\mathrm{H}\end{array}$ & $\begin{array}{l}.797 \\
.672 \\
.125\end{array}$ & $\begin{array}{r}.656 \\
.734 \\
-.078\end{array}$ & $\begin{array}{l}.750 \\
.703 \\
.047\end{array}$ & $\begin{array}{l}.781 \\
.656 \\
.125\end{array}$ & $\begin{array}{r}.688 \\
.750 \\
-.062\end{array}$ & $\begin{array}{l}.734 \\
.688 \\
.046\end{array}$ & $\begin{array}{l}.750 \\
.750 \\
.000\end{array}$ & $\begin{array}{l}.737 \\
.708 \\
.029\end{array}$ \\
\hline
\end{tabular}

distributions of the LEDs overlapped at the diffusing surface, so that switching from one to the other displaced the brightness peak by $3 \mathrm{~mm}$. The detectability of this displacement (the "probe signal") was highly dependent on the intensity of the probe LEDs; therefore, intensity was adjusted for each $S$ to produce probe-movement signals that were in the midthreshold region. The values chosen by this procedure were of the order of $.001 \mathrm{~cd}$. The Ss were unable to detect the probe displacement as a change in static location; to prevent them from doing so was one of the reasons for locating the probes off-center. Strictly speaking, perceived movement of the probe was itself "apparent" rather than "real," but the distinction is almost certainly inconsequential for a displacement through only 4 min of visual angle.

A dimly illuminated LED in the middle of the display served as a fixation point.

Timing and switching operations were done by a bank of six solid-state timers (crystal-controlled preset pulse counters with better than millisecond accuracy) constructed at the University of Oregon from kits supplied by the Southwest Technical Products Corporation.

\section{Procedure}

As noted, the Ss quickly learned to exercise almost perfect voluntary control over the path of the apparent movement, i.e., to see either vertical or horizontal movement as instructed from trial to trial. Horizontal movement appeared to pass through or over the probes; vertical movement did not. Thus, the major independent variable of the study was the $S$ 's internal representation of the movement (as determined by instructions) with everything constant at the receptor level. Masking specific to the path of the movement would be indicated by poorer detection of the probe signal during horizontal movement than during vertical movement. (Kolers determined by means of a control experiment that his second light had a backward masking effect on the probe flash, independent of the first light or apparent movement. We know that nonspecific masking likewise occurred in the present study, since a probe signal that was at threshold under the experimental conditions was clearly perceptible when the main lights were kept off altogether.)

On every trial, the $\mathrm{S}$ was told whether to see horizontal or vertical movement, whereupon the main lights were alternated through four complete cycles with a long flash duration, $600 \mathrm{~m} \mathrm{sec}$, and a negligible ISI of $1 \mathrm{msec}$, the minimum that the timers allowed. The illusion of oscillatory movement was excellent under these conditions. Within $120 \mathrm{msec}$ before or after the final transition, either the upper or the lower probe was displaced. The task of the $S$ (who of course knew, within narrow limits, when to expect the probe signal) was to tell whether the upper or the lower probe moved, and whether to the right or to the left.

The time at which the probe signal in fact occurred, relative to the final switching of the main lights, was varied over the following values: $-120,-60,-30,0,+30,+60$, and $+120 \mathrm{~m} \mathrm{sec}$. The conditions (1) horizontal vs vertical apparent movement,
(2) upper vs lower probe, (3) left vs right movement of probe, and (4) time of probe signal were varied orthogonally. The 56 combinations of these variables were employed twice in each of eight sessions per $S$, in a random order that was reversed from the first to the second half of the session.

Before the experiment proper, the Ss were given adequate practice in controlling the direction of the apparent movement, and enough experimental trials were run to make possible the adjustment of probe intensity to the threshold region for probe movement (ideally, $75 \%$ correct identification of the probe that moved). The $\mathrm{Ss}$ tended to improve in the course of the experiment, and further small adjustments of probe intensity were made on several occasions to counteract this tendency, but always between sessions, so that all conditions were affected equally.

\section{RESULTS}

Our most stable measure of detectability is the proportion of trials on which the $S$ correctly identified both the position of the probe that moved and the direction in which it moved; by pure guessing this would be .25 , and it is evident in Table 1 that we were working in just about the middle of the region of uncertainty. The proportions shown for the various probe times and directions of apparent movement are based on 64 trials; the means, on 448 trials.

Whatever our misgivings about the earlier experiment of Kolers, our results show no more evidence of masking than his did. Averaged over all probe times, S D's accuracy was exactly the same for the two paths of apparent movement, and the difference between S I's overall proportions is slightly less than its standard error. The two greatest differences (for S I at -120 and $0 \mathrm{msec}$ ) are .125 , but this value, considered in isolation, is only 1.6 times its standard error; moreover, the two cases are separated by values that have a negative average.

Breakdown of data by variables collapsed in Table 1 , including direction of probe movement, does nothing to change the totally negative picture, but does reveal some consistent biases: e.g., both Ss were more willing to report probe movement toward the second and closer light than toward the first and more distant one (regardless of other conditions), and S D showed further tendencies to attribute the direction of the apparent movement to the probe on horizontal trials, and to be 
correct more often on the lower probe than on the upper one.

\section{DISCUSSION}

It is a fair assumption that we would have obtained positive results if either movement masking or brightness masking had occurred, since small changes in probe intensity markedly affected the detectibility of probe movement. The negative results that we did obtain make it unnecessary to pursue this distinction, or to investigate the possibility of an interaction between probe time and position of the probe along the movement path.

It is conceivable that the range of our probe times was insufficient, but unlikely in view of what is known about the time scale on which apparent movement occurs. In some preliminary experiments, we tried in various ways to time or track the course of apparent movement. For example, we found that reaction times to the beginning of movement vs the end of movement (or to the offset of the first light vs the onset of the second; the form of the question made no difference) consistently differed by some 40 or $50 \mathrm{msec}$, although the transition was practically instantaneous in physical time. The interpretation of this difference is not unequivocal, however; it might indicate merely that attention was concentrated on the first rather than the second light at the time of the transition.
Although the demonstration of masking by apparent movement (particularly if the point of maximum masking along the path could be shown to covary with the time of the probe) would strongly indicate that the movement is subserved by a process that in fact moves through a representational medium, the absence of masking does not provide any symmetrically compelling disconfirmation of this hypothesis. Exactly the same amount of information about the probe entered the nervous system regardless of the apparent movement path-the experiment was carefully arranged to ensure this-and it is entirely possible that the $S$ was able to tap off this information at a low level, and evaluate it in a channel that bypassed the medium in which apparent movement occurred. The Ss' accounts of their subjective experiences were consistent with this possibility; likewise, when they were asked to specify when the probe signal occurred, relative to the course of the apparent movement, their judgments were erratic and uncertain, and showed surprisingly little covariation with actual probe times over the $240-\mathrm{msec}$ range.

\section{REFERENCES}

Attneave, F., \& Block, G. A pparent movement in tridimensional space. Perception \& Psychophysics, 1973, 13, 301-307.

Kolers, P. A. Some differences between real and apparent visual movem ent. Vision Research, 1963, 3, 191-206.

(Received for publication December 17, 1973; revision accepted A pril 8, 1974) 